\title{
Psychometric properties of the new Persian version of the Geriatric Anxiety Inventory (GAl-PV) and its short form (GAI -PV-SF)
}

Mohsen Shati

Iran University of Medical Sciences

Seyede salehe mortazavi ( $\square$ salehe.mortazavi@gmail.com )

Seyed Kazem Malakouti

Iran University of Medical Sciences

Shiva ASCEND Center for Biomedical Research Mehravaran

ASCEND Center for Biomedica research

Ali Norouzi

Tehran University of Medical Sciences

Nancy A. Pachana

University of Queensland

Research article

Keywords: Geriatric Anxiety Inventory (GAl (, aged, psychometric property, validity, reliability

Posted Date: October 18th, 2019

DOl: https://doi.org/10.21203/rs.2.16187/v1

License: (9) This work is licensed under a Creative Commons Attribution 4.0 International License.

Read Full License 


\section{Abstract}

Background The aim of this research was to analyze the psychometric properties of the Persian version of the Geriatric Anxiety Inventory (GAI-PV) and its short form (GAI-PV-SF) in a sample of older adults in Iran.

Methods In this cross-sectional study, a sample of 150 community-dwelling and a psychogeriatric sample of 48 adults older than 60 years completed the GAI-PV and GAI-PV-SF, the anxiety sub-scale of the General Health Questionnaire (GHQ-28), the Geriatric Depression Scale (GDS-15), and the Structured Clinical Interview for DSM-IV (SCID-I).

Results Both the GAI-PV and GAI-PV-SF exhibited excellent internal consistency and desirable concurrent validity against GHQ-28 and GDS-15. The optimal cutpoint score to detect current generalized anxiety disorder (GAD) was 10/11 and 13/14 for GAI-PV in the community-dwelling and psychogeriatric samples, respectively, and 3/4 for GAI-PV-SF in both study samples. A good test-retest reliability and a single-factor structure were also demonstrated.

Conclusion Sound psychometric properties of the GAI-PV in both subsamples suggest that the instrument could be used successfully as an accurate screening instrument in the elderly Iranian population.

\section{Introduction}

Mental illnesses, such as depression and anxiety, are health-related issues that need to be identified and addressed in elderly populations [1]. Despite its influence on the functional status and quality of life of older adults, anxiety remains undiagnosed or untreated in many cases, and receives less attention than depression among the elderly; this can be due to the lower prevalence of anxiety in older adults compared to other age groups [2-4]. The importance of anxiety lies in the fact that it frequently manifests with somatic symptoms, and their overlap with those of age-related diseases can make the diagnosis more difficult and lead to under-reporting of anxiety in the elderly [5].

Studies have reported different prevalence rates of anxiety disorders among the elderly. The discrepancy can be due to different research methodologies, duration criteria (e.g. one month, six months, and one year), diagnostic tools and criteria, and true differences in prevalence rates in certain populations [6-8].

Various instruments are available for the diagnosis of anxiety in the elderly. The Beck Anxiety Inventory (BAI) is one such tool for which normative data are available for elderly samples [9]. For tools such as the Adult Manifest Anxiety Scale, an elderly version has been created by making appropriate modifications to the original version [10]. There are also certain less common instruments, such as the Short Anxiety Screening Test (SAST), which have been designed for the elderly [11]. However, many of these evaluation tools, even those specifically designed for the elderly, have certain psychometric and clinical limitations. 
The Geriatric Anxiety Inventory (GAl) was created by Pachana et al. in 2007 in Australia and can be used as a screening instrument for dimensional anxiety in older adults. Originally, the instrument comprised 60 items (de novo and by reference to 25 existing scales), and after testing the psychometric properties of these 60 items, a 20-item instrument was developed [4]. The 20-item GAl is a brief self-report or nurseadministered scale which helps avoid respondent fatigue and can be used in various settings. The instrument has dichotomous questions (I agree / I disagree), and thus, can be suitable for individuals with low literacy levels or low cognitive ability. By minimizing the emphasis on somatic symptoms, it also limits the potential overlap with physical diseases [4].

The GAl is not intended for the classification of anxiety disorders, but rather examines the overall presence or absence of anxiety symptoms in the elderly individual. A higher score is indicative of more severe symptoms, and the highest possible score is 20 . Studies on the GAl suggest that a score of 10/11 is the optimum cutpoint with $84 \%$ sensitivity and $75 \%$ specificity [4]. Other studies assessing the psychometric properties of this instrument have arrived at similar results [3]. Byrne and Pachana have developed a short version of the instrument which contains 5 (items number $1,6,8,10$, and 11) of the original 20 items, and it has been found suitable for the detection of DSM-IV generalized anxiety disorder (GAD) in older populations using a cutpoint score of 3 which provides a sensitivity and specificity of $75 \%$ and $87 \%$, respectively [12].

In 2019, Bandari et al. conducted a psychometric analysis of a Persian version of the GAl in an Iranian sample. Despite a relatively large ample size (720 cases), the study participants were selected only from those referring to a primary health care center for routine services. Moreover, they did not use a gold standard, and thus, no cutpoint was provided; lack of a gold standard for comparison limits the ability to assess the screening and diagnostic value and estimating the sensitivity and specificity or the likelihood ratio [13]. Therefore, the present study was designed in collaboration with the developer of the original survey to prepare a Persian version of the GAI (GAI-PV) and its short version (GAI-PV-SF) and test their psychometric properties in elderly Iranian samples.

\section{Methods}

\section{Sample}

Study participants included 150 community-dwelling older adults recruited from the general population through convenience sampling at public places such as parks, community centers, neighborhood markets, and mosques. An additional 48 elderly patients were included as a psychogeriatric sample among patients referring to the Psychology Clinic at Rasool Akram Hospital and did not have clinically significant cognitive impairment as judged by the psychologist. All selected individuals were 60 years of age or older and native speakers of Persian.

\section{Procedure}


In developing the GAI-PV, first the forward translation to Persian was done by a team of 3 psychologists with expertise in the field of geriatric mental health. The translated version of the questionnaire was examined by the researchers for its semantic and content equivalence and acceptability, and necessary revisions were made. Backward translation to English was then done by another near-native translator with knowledge and experience in the field of psychologic disorders. All items with translations identical to the original questionnaire were retained; the rest were discussed with the first translators, and an iterative process of forward and backward translation was conducted until all items in the backward translation fully matched the original scale.

To promote face validity, the developed GAI-PV underwent panel review by a sample of 10 healthy monolingual older adults. After reading and hearing the questions, the participants stated that they found the questionnaire very easy. Any part that appeared difficult to comprehend was revised using feedback from these participants and the research team.

To examine the internal consistency, we determined the Cronbach's alpha of the full and short versions of the translated instrument. This measure was calculated for the community-dwelling and the psychogeriatric groups separately.

In the next stage, concurrent validity was tested using the anxiety subscale of the GHQ-28 and the GDS. For this purpose, the agreement of results obtained from GAI-PV and its short version with those from the GHQ-28 and the GDS were examined by computing Pearson correlation coefficients in the communitydwelling and psychogeriatric groups of the study. A subgroup of 40 participants was selected from the community-dwelling group to examine the test-retest validity of the questionnaires, and the interview process was repeated 2 weeks later. Differences between the test and retest were used as a measure for this type of validity.

Finally, optimal cut points were determined using the receiver operating characteristic (ROC) curve. For this purpose, the point with the highest sensitivity and specificity to discriminate between those with and without generalized anxiety disorder (GAD) was determined. At this stage, the Structured Clinical Interview (SCID-I) was considered the gold standard method for the diagnosis of GAD. All data collected so far were analyzed using the SPSS statistical software (SPSS Inc.).

In line with previous studies with this instrument in other countries, the construct validity was tested through confirmatory factor analysis (CFA) after conducting a descriptive analysis to detect outliers and missing data. CFA was done using the maximum likelihood estimation method. First a single factor model was described, and the factor loadings of each item were computed along with statistical significance tests. In the next step, results were reviewed, and items with a factor loading value of 0.3 or more and a t-value more than 1.96 were retained.

Model fit in this study was assessed using the parameters proposed by Hu and Bentler [14]. These included the root mean square error of approximation (RMSEA), the standardized root mean square residual (SRMR), the comparative fit index (CFI), the goodness of fit index (GFI), the adjusted goodness of 
fit index (AGFI), Normed Fit Index (NFI) and the Non- Normed Fit Index (NNFI).For RMSEA and SRMR, which are known as relative fit measures, values less than 0.05 are interpreted as perfect fit, 0.05 to 0.08 is acceptable, and models with values more than 0.08 are rejected. For $\mathrm{GFI}, \mathrm{AGFI}, \mathrm{NFI}$ and NNFI values more than 0.9 were considered a good fit. All factor analyses were conducted using version 8.8 of the LISREL software (Scientific Software International, Inc.).

\section{Instruments}

For illiterate individuals, all surveys items, except SCID-I, were fully read to the participants by an interviewer, and in other cases, it was self-administered. Survey administration was completed in a space approved by the participants.

\section{Sociodemographic characteristics}

In this study, demographics were collected using a survey to record age, gender, marital status, educational attainment, occupation, medical history, and family history of psychological disorders.

\section{General Health Questionnaire (GHQ-28)}

The GHQ-28 instrument was developed by Goldberg et al. in 1979 to assess mental health through primary health care screening programs. The 28 items form 4 subscales of 7 items each: anxiety, somatization, social dysfunction, and depression. Scores are calculated for each subscale and the total [15]. In this study, only the anxiety scale of the GHQ-28, which has been validated for the general and elderly populations in Iran, was used to assess concurrent validity. Responses were recoded to zero for values of 1 and 2, and to 1 for values of 3 and 4 , and a total score of 2 or more (out of a maximum of 7 ) was used as the cutpoint to identify suspected cases [16].

\section{Geriatric depression scale (GDS)}

The GDS is a 30-item questionnaire designed to assess depression in the elderly [17]. The short form (GDS-15) has been tested for validity and reliability in older adults in Iran [18]. In this study, we examined the agreement between scores obtained from GAI and GDS-15.

\section{Structured Clinical Interview for DSM-IV (SCID)}

SCID is a semi-structured interview for the diagnosis of DSM-IV disorders. This instrument is the most widely used standard diagnostic interview in psychologic studies [19]. There are two versions: SCID-I which assesses axis-I psychological disorders and SCID-II which assesses axis-II personality disorders [20]. The validity and reliability of this instrument has been examined in Iran by Sharifi et al. [2]. In this 
study, we used this instrument as the gold standard for the diagnosis of generalized anxiety disorder, determine the best cutoff, and compute the sensitivity and specificity of GAl.

\section{Ethical considerations}

The proposal of this study was reviewed and approved by the Ethics Committee of Iran University of Medical Sciences under number IR.IUMS.REC1393.92-03-121-23143. The methods and procedures of the study were explained to participants, and they all signed informed consents before being enrolled. Participating individuals were assured of confidentiality and were given the choice to withdraw from the study at any stage they wished. Those who were identified as suspect cases of anxiety were referred to a specialist for further diagnostic measures and treatment.

\section{Results}

\section{Descriptive statistics}

As presented in Table 1, the majority of participants were married men in the 60-74 age group. In the community-dwelling and psychogeriatric groups, respectively $2.0 \%$ and $4.2 \%$ were fully employed, while $75.3 \%$ and $60.4 \%$ were retired pensioners.

Mean ( \pm standard deviation) GAI-PV and GAI-SF-PV scores were respectively $4.93 \pm 5.23$ and $1.56 \pm 1.72$ in the community-dwelling group and $14.86 \pm 4.76$ and $3.95 \pm 1.41$ in the psychogeriatric group. Mean scores did not significantly correlate with age, gender, number of children, marital status, education, or occupation in either study group. About one-third (33.8) of the community-dwelling participants were diagnosed as anxiety suspects based on the GHQ-28 results; this rate was about three times higher (89.4) in the psychogeriatric group. The proportion of depression suspects was also about 4 times higher in the psychogeriatric group compared to community-dwellers (72.1\% versus $17.9 \%)$. Definitive cases of GAD, who were diagnosed using SCID-I, constituted $2.0 \%$ of the community-dwellers and $66.7 \%$ of the psychogeriatric group.

\section{Validity}

To confirm face validity, the research team compared the original version with the backward translation and found no significant discrepancy between them. However, the monolingual review panel, who tested the questionnaire for this very purpose, reported that item number 7 was confusing. Accordingly, it was decided that the idiom "have butterflies in my stomach" needed to be rephrased to an equivalent Persian idiom resolve comprehension difficulty.

Concurrent validity was assessed by comparing the agreement between the 20-item GAI-PV and 5-item GAI-SF-PV with the anxiety subscale of the GHQ-28 and the GDS. According to the results (Table 3), the 
agreement between GAI-PV and GAI-SF-PV was very high, and the agreement levels between the results obtained from these two instruments and the anxiety scale of the GHQ-28 and GDS were acceptable.

\section{Reliability}

Internal consistency for the GAI-PV was measured through the Cronbach's 4 which was generally acceptable for the community-dwelling and psychogeriatric groups (0.91 and 0.80 , respectively). The corrected item-total correlation values for items 18,12 , and 13 were $0.16,0.24$, and 0.40 , respectively, and higher than 0.5 for all other items. For GAI-SF-PV, the Cronbach's $\nabla$ was 0.8 for the community-dwelling group and 0.74 for the psychogeriatric group. The agreement between pre-test and post-test scores obtained from the GAI-PV and the GAI-SF-PV was 0.96 and 0.88 , respectively and the small difference between pre-test and post-test scores was not statistically significant (P-value $=0.78$ and 0.23 ).

\section{The Cutpoint score}

The area under the curve for GAI-PV was 0.89 (95\% confidence interval $(\mathrm{Cl})$ : $0.82-0.97)$ in the communitydwelling group and 0.83 (95\% Cl: 0.69-0.97) in the psychogeriatric group. The optimal cutpoint compared to SCID-I was 10/11 for the community-dwelling group with $100 \%$ sensitivity and $80.6 \%$ specificity and $13 / 14$ for the psychogeriatric group with $97 \%$ sensitivity and $62 \%$ specificity.

The likelihood ratio for a negative test result ( $\left(\mathrm{R}^{-}\right)$was 0.04 in the psychogeriatric group and 0 in the community-dwelling group; this means there was a large change in the pre- to- posttest odds of disease. The likelihood ratio for a positive test result $\left(\mathrm{LR}^{+}\right)$was 2.50 in the psychogeriatric group and 5.15 in the community-dwelling group.

After excluding 3 somatic items (7, 12, and 18), the optimal cutpoint for the GAI-PV for the communitydwelling and psychogeriatric groups was $10 / 11$ and $11 / 12$, respectively; the areas under the curve were respectively $91 \%$ and $82 \%$, and both were statistically significant.

ROC analysis was conducted for the GAI-SF as well. The area under the curve was 0.86 (95\% Cl: 0.72$0.99)$ in the psychogeriatric group and 0.89 (95\% Cl: $0.82-0.96)$ in the community-dwelling group. The optimal cutpoint compared to SCID was 3/4 for the psychogeriatric group with $100 \%$ sensitivity and $64 \%$ specificity, and $3 / 4$ for the community-dwelling group with $100 \%$ sensitivity and $81.6 \%$ specificity. LR Ras $^{-}$was 0 in both the psychogeriatric and community-dwelling samples and LR ${ }^{+}$was 2.70 in the psychogeriatric sample and 5.43 in the community dwelling sample.

\section{Confirmatory factor analysis}

The initial CFA model for GI-PV was drawn with 20 items with the assumption of a single factor. As illustrated in figure 1, except for items 12 and 18, all items had acceptable and significant factor loading. 
The goodness of fit indices for the initial model was not acceptable $(\chi 2=448.02, P=0.00, R M S E A=0.09$, $\mathrm{RMR}=0.01, \mathrm{NFI}=0.93, \mathrm{NNFI}=0.95, \mathrm{CFI}=0.95, \mathrm{GFI}=0.8, \mathrm{AGFI}=0.75)$.

Therefore, to arrive at acceptable goodness of fit indices, two rounds of modification were done. In the first modification, item number 18 was excluded because of poor factor loading (0.16) and a T-value of 1.86. The next modification, as suggested by the LISREL software, was drawn on the strong covariance relationship between variance errors in items 10 and 13 . This resulted in very suitable goodness of fit indices for the model $(X 2=304.66, \mathrm{P}=0.00, \mathrm{RMSEA}=0.07, \mathrm{RMR}=0.01, \mathrm{NFI}=0.96, \mathrm{NNFI}=0.98, \mathrm{CFI}=$ $0.98, \mathrm{GFI}=0.85, \mathrm{AGFI}=0.81$ ), and eventually, the final single-factor model with 19 items was approved for this construct. Although item number 12 had weak factor loading, it was retained given the significance level>1.96. (T-value $=2.93)$ (figure 2).

\section{Discussion}

The aim of this study was to determine the psychometric properties of the Persian version of GAl and its short form in two separate groups of community-dwelling and psychogeriatric samples. Results of the analyses, including internal consistency, test-retest, concurrent validity and ROC, which were mostly in agreement with other similar studies, indicated that both GAI-PV and GAI-SF-PV are acceptably valid and reliable, and they can be used for clinical and research purposes to identify cases of GAD in different settings (general public and in-patient clinics).

After the forward and backward translation of the scale, certain items appeared to be different from the original questionnaire [4]. Comprehension difficulty with certain items was experienced in translations to other languages as well. A common issue was item number 7, which as mentioned, was completely changed in the Persian version. This item created a similar challenge in translating the scale to Portuguese, Spanish, Chinese, and Norwegian; the phrase was not translated word for word because it was not relevant to the native culture, and instead, a conceptually equal phrase was used to make it comprehensible [21-25]. For example, the item was modified to "I feel pressure in my chest" in the Portuguese version and to "I feel there is something like ants in my stomach" in the Spanish version [22, 23]. For the same reason of cultural differences, a similar challenge was experienced with other items in some other languages. For example, Mirada-Castillo et al. who conducted a similar study in Chile, reported that items 9,16 , and 19 were modified in terms of the idioms that were used [26].

As indicated by a Cronbach's alpha of over $80 \%$, the internal consistency of GAI-PV was as acceptably good as that reported in similar studies in other countries [3]. The estimates for GAI-PV and GAI-SF-PV were similar to those of the French-Canadian version, and quite comparable to the Cronbach's alpha generated from the studies in Spain and Portugal, the study by Pachana, and the study by MirandaCastillo et al. $[4,22,23,26]$. Similar to the original version, the corrected item-total correlation was higher than 0.5 for 17 of the 20 items in GAI-PV; this is also in line with the study of the Chinese version [24].The lower corrected item-total correlation for items 12 and 18 could be due to their relevance to somatic symptoms of anxiety. Given the higher prevalence of chronic comorbidities in older age groups, it may be 
difficult to differentiate somatic symptoms related to anxiety from those related to physical diseases, and symptoms cannot always be attributed to anxiety; this could explain the weak association between somatic symptoms and anxiety. Previous studies have mentioned this issue with items 7, 12, and 18 as well [3]. Another reason for the lower association could be the comprehensibility of these items which leads to modified contents during the process of forward and backward translation [3].

The test-retest reliability for the long version of GAI-PV was excellent (Spearman correlation coefficient of 0.97), and these results are in agreement with those presented by Pachana et al. in the original development paper [4]. The reliability estimates with the Italian and Australian versions were lower than the original and current Persian version, however, they were still in the acceptable range $(0.86$ and 0.76 , respectively) $[3,4,27]$. The test-retest reliability was very good for the GAI-SF-PV as well $(0.88, p<0.0001)$. Test-retest reliability is not mentioned in the study by Bandari et al. [13].

The CFA in the present study found a unidimensional model; this is in line with the original study [4] and some other similar studies $[3,21,24,26,28]$. Nonetheless, as presented in the results, items 12 and 18 had low factor loading, and excluding item 18 appeared to improve the goodness of fit. This is in agreement to the study by Molde et al. who performed a cross-national analysis of the psychometric properties of the GAl using data from samples in 10 different countries; although they concluded that the instrument can be more unidimensional without items 7,12 , and 18 , they suggested that these candidate items be retained to avoid decreased content validity [3]. The weak factor loading of these items appears to be connected to study participants' interpretation and perception of their contents. In other words, the items contained colloquial phrases and idiomatic expressions relevant to a given culture, and a verbatim translation cannot convey their true meaning. Therefore, we recommend using the 20 -item version of the GAI-PV without excluding any items, provided that it is adapted to the local culture and language by using culturally appropriate phrases and idioms rather than verbatim translation. For example, "feeling chaos in one's stomach" or "having laundry in one's stomach" are equivalent phrases for "having butterflies in one's stomach".

Some studies have considered multiple domains (2 to 4) for the GAl questionnaire [3, 23]. In the study of Bandari et al, the GAl initially underwent exploratory factor analysis, and three factors were extracted. This three-factor structure was then confirmed through CFA [13]. Comparison between studies that have used a single-factor structure with studies that have used as a three-factor version does not show a considerable difference between the levels of total variance explained. For example, Champagne et al. showed that a single-factor questionnaire explains $60 \%$ of the variance [28]. While a higher rate was expected with the 3-factor version, the study by Bandari et al. indicated that the 3-factor also explained about $59.5 \%$ of the variance [13]. Therefore, we assumed a single-factor structure for GAI in our study and refrained from an exploratory factor analysis. CFA also showed that, with minor adjustments, this tool has a suitable and acceptable single-factor structure and results are comparable to most other versions in other countries $[3,21,24,26,28]$. 
Another point worth mentioning is that Bandari et al. have made no mention why verbatim translation of the items of concern were used without any modification in the choice of words [13]. While the authors claim to have incorporated cultural considerations in translating the instrument, such considerations are not found in the instrument appended to the paper. The instrument contains item 7 , for example, without any change to its original content (I often feel like I have butterflies in my stomach), and although it has no clear meaning in the Iranian culture, it appeared as one of the strongest items in the exploratory factor analysis (factor loading of 0.77 ). In the present study, this item was translated to "I often feel chaos in my stomach", and factor analysis indicated a very strong factor loading of 0.83 .

Bandari et al. also used item 18 without changing its content (I sometimes feel a great knot in my stomach), and CFA indicated a factor loading of 0.6 and variance error of 0.6 [13]. In this case also, the verbatim translation of "feeling knots in the stomach" lacks any meaning in Persian, and it is not clear how such a good load is achieved without considering this important issue and making necessary changes. In our study, this item was translated to "sometimes I feel something is stuck in my throat", and it was excluded due to a poor factor loading of 0.14 . Therefore, although we attempted to use conceptual translation for the items that appeared to require cultural/linguistic adjustments in order to improve their validity, our results with items 12 and 18 were not quite as good.

The best cutpoint for the detection of GAD with GAI-PV compared to SCID was 13/14 in the psychogeriatric group and 10/11 in the community-dwelling sample; with GAl-SF-PV, the best cutpoint was $3 / 4$ in both groups. The area under the curve was favorable with both forms of the instrument in both groups of the study. In terms of sensitivity and specificity of the cutpoints, both the long and short versions of GAI-PV demonstrated very high sensitivity, and are thus appropriate tools for screening generalized anxiety disorder in the community-dwelling and psychogeriatric samples, and a score below the cutpoint is sufficient to rule out disease $\left(\mathrm{LR}^{-}<0.04\right)$. Of course, given the acceptable level of specificity of the cutpoints, the test can be used to confirm the diagnosis of GAD in community-dwelling populations, and a score that exceeds the cutpoint is associated with a 5 -fold increase in the odds of $\mathrm{GAD}\left(\mathrm{LR}^{+}>5\right)$.

Studies in other countries have arrived at different cutpoints for each version $[23,26,28]$. Pachana et al. concluded that a cutpoint of $10 / 11$ is the best for detecting GAD in psychogeriatric samples [4]. Ribeiro et al. suggested a cutpoint of $8 / 9$ for detecting severe anxiety symptoms with the Portuguese version of this instrument, but they could not estimate an optimal cutpoint for detecting GAD [22]. In the study by Pachana and Byrne, the optimal cutpoint with the short form of this tool for community-dwelling elderly women was 3 [12].

The higher cutpoint estimated with the Persian version may be due to cultural differences between elderly Iranian and those in other countries. Perceptions of healthy and unhealthy older men appear to differ in developing eastern societies compared to developed communities. Fear of being ignored in a society where social support systems lack enough resources to address the physical and psychological problems of the elderly [29] can lead to exaggerated symptoms with the intention to seek attention. Multiple studies

Page 10/19 
have pointed to higher prevalence rates of somatic complaints among Iranians instead of expressing psychological symptoms. Such behaviors have been attributed to fear of appearing powerless and being judged, and the concept that somatic and physical symptoms are more acceptable and carry less stigma compared to psychological complaints [30,31]. It seems that the high cutpoint of GAI-PV is due to items 7,12 , and 18 about somatic symptoms, as evidenced by the lower cutpoint in the psychogeriatric sample after removing these items. Balsamo et al. who conducted a review study also concluded that adding somatic items to anxiety questionnaires for the elderly leads to inflated scores, which is consistent with the results obtained in this study [31].

The strong and significant correlation of GAI-PV and GAI-PV-SF results in both community-dwelling and psychogeriatric groups with the anxiety subscale of the GHQ-28 and the moderate correlation with the GDS, which is used to assess depression, are indicative of good results in line with other similar studies and desirable concurrent validity for these two instruments. However, different tools have been used to measure concurrent validity in different studies, and an important factor in selecting these tools is going through the localization process in these countries $[4,23,24,26,27]$. In the study by Bandari et al. concurrent validity was not tested using anxiety and depression scales designed for the elderly. Instead, they used the SF-36 General Health Survey; the cognitive and arousal subscales of their version of the GAl showed a correlation coefficient less than 0.5 with the mental health subscale of the SF- 36 and a correlation coefficient of 0.27 with its somatic subscale [13].

Based on our results, GAI-PV and GAI-PV-SF were strongly correlated, and this is in agreement with results of previous studies. Given the brevity and acceptable accuracy of GAI-PV-SF, it could be used in research and for the assessment of individuals referring to health care settings, because usually other surveys and questionnaires are also administered in these cases, and using the short form of this questionnaire can help save time, prevent survey fatigue, and improve accuracy [4].

\section{Conclusions}

The desirable reliability and validity obtained in this study implies that both the complete and short forms of this questionnaire can be successfully used as a suitable screening tool in clinical settings as well as in epidemiological studies that aim at assessing anxiety disorders in older adults.

\section{Abbreviations}

AGFl: adjusted goodness of fit index, BAl: Beck Anxiety Inventory, CFA: confirmatory factor analysis, GAl: Geriatric Anxiety Inventory, GAI-PV: Geriatric Anxiety Inventory Persian version, GAI-PV-SF: Geriatric Anxiety Inventory Persian version Short Form, GAD: Generalized Anxiety Disorder, GDS: Geriatric Depression Scale, GHQ: General Health Questionnaire, GFI: goodness of fit index, NNFI: Non- Normed Fit Index, SAST: Short Anxiety Screening Test, SCID-I: Structured Clinical Interview for DSM-IV, SRMR: standardized root mean square residual, RMSEA: root mean square error of approximation 


\section{Declarations}

\section{Acknowledgements}

This research was funded by the Mental Health Research Center of Iran University of Medical Sciences. The authors wish to thank all study participants, especially the elderly, who collaborated with us in this project.

\section{Authors' contributions}

SSM and MS designed the study and performed the data analysis. SKM assisted in the study design and the data analysis. SM and AN interpreted the data and drafted the manuscript. All authors read and approved the final manuscript prior to submission.

\section{Funding}

This work was supported by the Mental Health Research Center of Iran University of Medical Sciences [grant number 92-03-121-23143]. Our funders had no role in the design or execution of the study, data analysis or interpretation, or writing of this manuscript.

\section{Availability of data and materials}

Data supporting the findings are available on request from the first author (mortazavi.ss@iums.ac.ir), not including personal identifiers based on our informed consent.

\section{Ethics approval and consent to participate}

The proposal of this study was reviewed and approved by the Ethics Committee of Iran University of Medical Sciences under number IR.IUMS.REC1393.92-03-121-23143. All participants were informed about the study and only those providing a written informed consent were enrolled in the study.

\section{Consent for publication}

Not applicable.

\section{Competing interest}


None.

\section{References}

1.Byrne GJ, Pachana NA: Anxiety and depression in the elderly: do we know any more? Current opinion in psychiatry. 2010; 23(6):504-509.

2.Sharifi V, Assadi SM, Mohammadi MR, Amini H, Kaviani H, Semnani Y, Shabani A, Shahrivar Z, DavariAshtiani R, Shooshtari MH: A persian translation of the structured clinical interview for diagnostic and statistical manual of mental disorders: psychometric properties. Comprehensive psychiatry. 2009; 50(1):86-91.

3.Molde H, Nordhus IH, Torsheim T, Engedal K, Bendixen AB, Byrne GJ, Márquez-González M, Losada A, Feng L, Ow EKT: A Cross-National Analysis of the Psychometric Properties of the Geriatric Anxiety Inventory. The Journals of Gerontology: Series B. 2019.

4.Pachana NA, Byrne GJ, Siddle H, Koloski N, Harley E, Arnold E: Development and validation of the Geriatric Anxiety Inventory. International Psychogeriatrics. 2007; 19:103-114.

5.Lang A, Stein M: Anxiety disorders. How to recognize and treat the medical symptoms of emotional illness. Geriatrics (Basel, Switzerland). 2001; 56(5):24-27, 31-24.

6.Ritchie K, Artero S, Beluche I, Ancelin M-L, Mann A, Dupuy A-M, Malafosse A, Boulenger J-P: Prevalence of DSM-IV psychiatric disorder in the French elderly population. The British Journal of Psychiatry. 2004; 184(2):147-152.

7.Mortazavi SS, Shati M, Ardebili HE, Mohammad K, Beni RD, Keshteli AH: Comparing the effects of group and home-based physical activity on mental health in the elderly. International journal of preventive medicine. 2013; 4(11):1282.

8.Kirmizioglu Y, Doğan O, Kuğu N, Akyüz G: Prevalence of anxiety disorders among elderly people. International Journal of Geriatric Psychiatry: A journal of the psychiatry of late life and allied sciences. 2009; 24(9):1026-1033.

9.Beck AT, Epstein N, Brown G, Steer RA: An inventory for measuring clinical anxiety: psychometric properties. Journal of consulting and clinical psychology. 1988; 56(6):893.

10.Lowe PA, Reynolds CR: Exploratory analyses of the latent structure of anxiety among older adults. Educational and Psychological Measurement. 2000; 60(1):100-116.

11.Sinoff G, Ore L, Zlotogorsky D, Tamir A: Short anxiety screening test-a brief instrument for detecting anxiety in the elderly. International journal of geriatric psychiatry. 1999; 14(12):1062-1071. 
12.Byrne GJ, Pachana NA: Development and validation of a short form of the Geriatric Anxiety Inventorythe GAI-SF. International Psychogeriatrics. 2011; 23(1):125-131.

13.Bandari R, Heravi-Karimooi M, Miremadi M, Mohebbi L, Montazeri A: The Iranian version of geriatric anxiety inventory (GAI-P): a validation study. Health and quality of life outcomes. 2019;17(1):118.

14.Hu L-T, Bentler PM: Evaluating model fit. 1995.

15.Goldberg DP, Hillier VF: A scaled version of the General Health Questionnaire. Psychological medicine. 1979; 9(1):139-145.

16.Noorbala A, Mohammad K: The validation of general health questionnaire-28 as a psychiatric screening tool. Hakim Research Journal. 2009; 11(4):47-53.

17.Sheikh JI, Yesavage JA: Geriatric Depression Scale (GDS): recent evidence and development of a shorter version. Clinical Gerontologist: The Journal of Aging and Mental Health. 1986.

18.Malakouti K, Fathollahi P, Mirabzadeh A, Salavati M, Kahani S: Validation of geriatric depression scale (GDS-15) in Iran. Research in Medicine. 2006; 30(4):361-369.

19.Mortazavi SS, Shati M, Malakouti SK, Mohammad K: Psychiatric comorbidities among Iranian elderly patients on methadone maintenance treatment. Archives of Iranian Medicine (AIM). 2015; 18(11).

20.Spitzer RL, Williams JB, Gibbon M, First MB: The structured clinical interview for DSM-III-R (SCID): I: history, rationale, and description. Archives of general psychiatry. 1992; 49(8):624-629.

21.Molde H, Hynninen K, Torsheim T, Bendixen A, Engedal K, Pachana N, Nordhus I: A Bifactor and item response analysis of the geriatric anxiety inventory. International psychogeriatrics. 2017; 29(10):16471656.

22.Ribeiro O, Paúl C, Simões MR, Firmino H: Portuguese version of the Geriatric Anxiety Inventory:

Transcultural adaptation and psychometric validation. Aging \& Mental Health. 2011; 15(6):742-748.

23.Márquez-González M, Losada A, Fernández-Fernández V, Pachana NA: Psychometric properties of the Spanish version of the Geriatric Anxiety Inventory. International Psychogeriatrics. 2012; 24(1):137-144.

24.Yan Y, Xin T, Wang D, Tang D: Application of the Geriatric Anxiety Inventory-Chinese Version (GAl-CV) to older people in Beijing communities. International psychogeriatrics. 2014; 26(3):517-523.

25.Pachana NA, Byrne GJ: The Geriatric Anxiety Inventory: international use and future directions. Australian Psychologist. 2012; 47(1):33-38.

26.Miranda-Castillo C, Contreras D, Garay K, Martínez P, León-Campos MO, Farhang M, Morán J, Fernández-Fernández V: Validation of the Geriatric Anxiety Inventory in Chilean older people. Archives of gerontology and geriatrics. 2019; 83:81-85. 
27.Byrne GJ, Pachana NA, Goncalves DC, Arnold E, King R, Keat Khoo S: Psychometric properties and health correlates of the Geriatric Anxiety Inventory in Australian community-residing older women. Aging \& Mental Health. 2010; 14(3):247-254.

28.Champagne A, Landreville P, Gosselin P, Carmichael P-H: Psychometric properties of the French Canadian version of the Geriatric Anxiety Inventory. Aging \& mental health. 2018; 22(1):40-45.

29.Noroozian M: The elderly population in iran: an ever growing concern in the health system. Iranian journal of psychiatry and behavioral sciences. 2012; 6(2):1.

30.McGoldrick M, Giordano J, Preto NG: Ethnicity and family therapy: Guilford Press. 2005.

31.Balsamo M, Cataldi F, Carlucci L, Fairfield B: Assessment of anxiety in older adults: a review of selfreport measures. Clinical interventions in aging. 2018; 13:573.

\section{Tables}

Table 1- Demographic characteristics of the community-dwelling and psychogeriatric group of the study

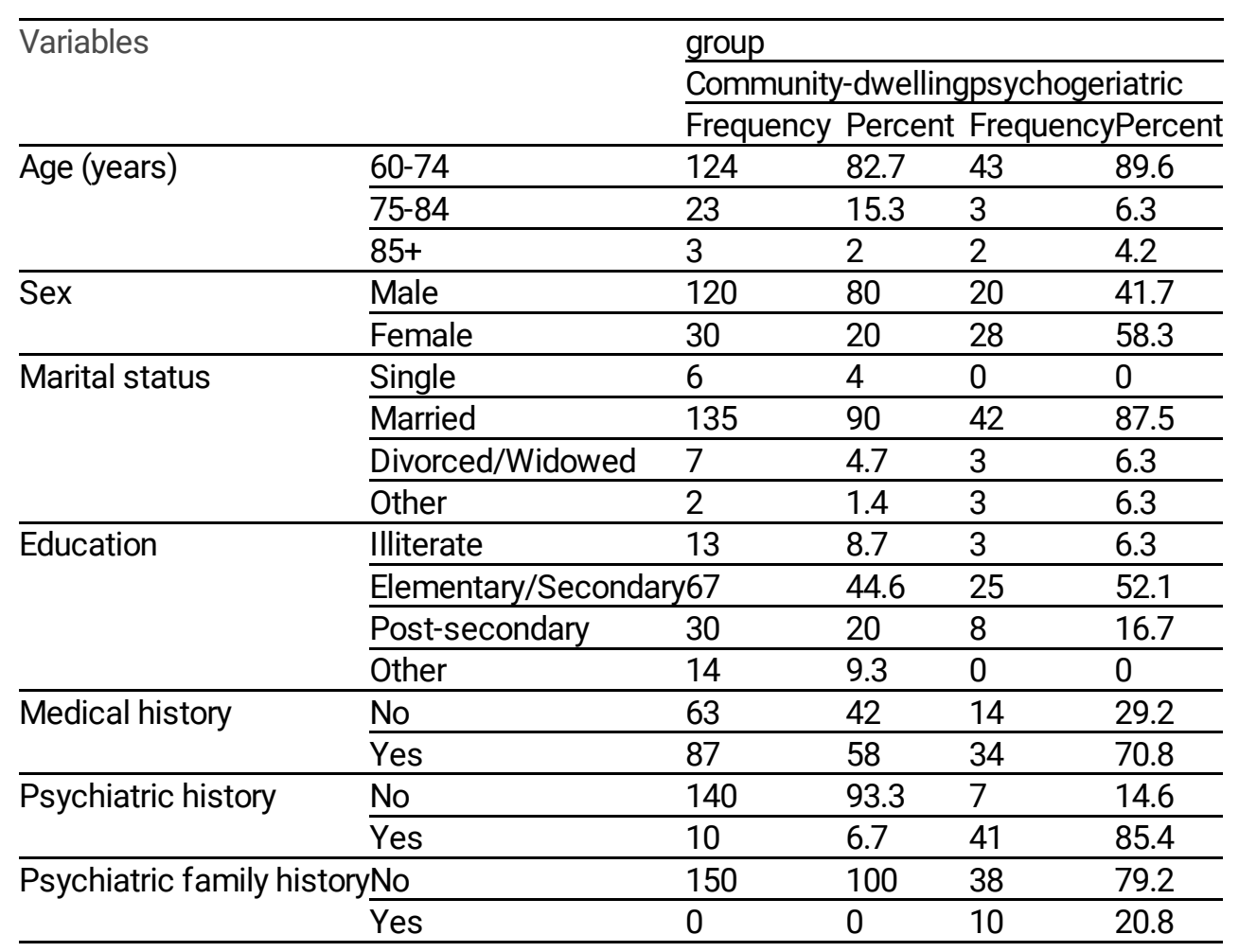

Table 2. Mean ( \pm standard deviation) scores with the GAI-PV and GAI-PV-SF, the anxiety sub-scale of the GHQ-28, and GDS-15 in the two study groups 


\begin{tabular}{lllll}
\hline \multirow{2}{*}{ InstrumentCommunity-dwelling groupPsychogeriatric group } \\
\cline { 2 - 5 } & Mean & SD & Mean & SD \\
\hline GAI-PV & 4.93 & 5.23 & 14.86 & 4.76 \\
\hline GAI-SF-PV 1.56 & 1.72 & 3.95 & 1.41 \\
\hline GHQ-28 & 1.54 & 2.03 & 5.27 & 2.10 \\
\hline GDS & 4.25 & 3.54 & 9.76 & 3.77 \\
\hline
\end{tabular}

Table 3. Spearman correlation coefficients between participants' scores in the GAI-PV, GAI-PV-SF, the anxiety sub-scale of the GHQ28 and GDS-15 in the two study groups

\begin{tabular}{|c|c|c|c|c|c|}
\hline \multirow{2}{*}{\multicolumn{2}{|c|}{$\begin{array}{l}\text { Group } \\
\text { Community-dwellingGAI-PV }\end{array}$}} & \multicolumn{4}{|c|}{ GAI-PVGHQ-28GAI-SF-PVGDS-15 } \\
\hline & & 1 & $0.68^{\star \star}$ & $0.90^{\star \star}$ & $0.65^{\star \star}$ \\
\hline & GAI-SF-P & $V_{0.90^{\star *}}$ & $0.69^{\star \star}$ & 1 & $0.62^{\star \star}$ \\
\hline \multirow[t]{2}{*}{ Psychogeriatric } & GAI-PV & 1 & $0.62^{\star \star}$ & $0.86^{* \star}$ & $0.50 \star \star$ \\
\hline & $\bar{G}$ & $0.86^{\star \star}$ & $0.63^{\star \star}$ & 1 & $0.52^{\star \star}$ \\
\hline
\end{tabular}

${ }^{* *}$ p- value $<0.001$

Figures 


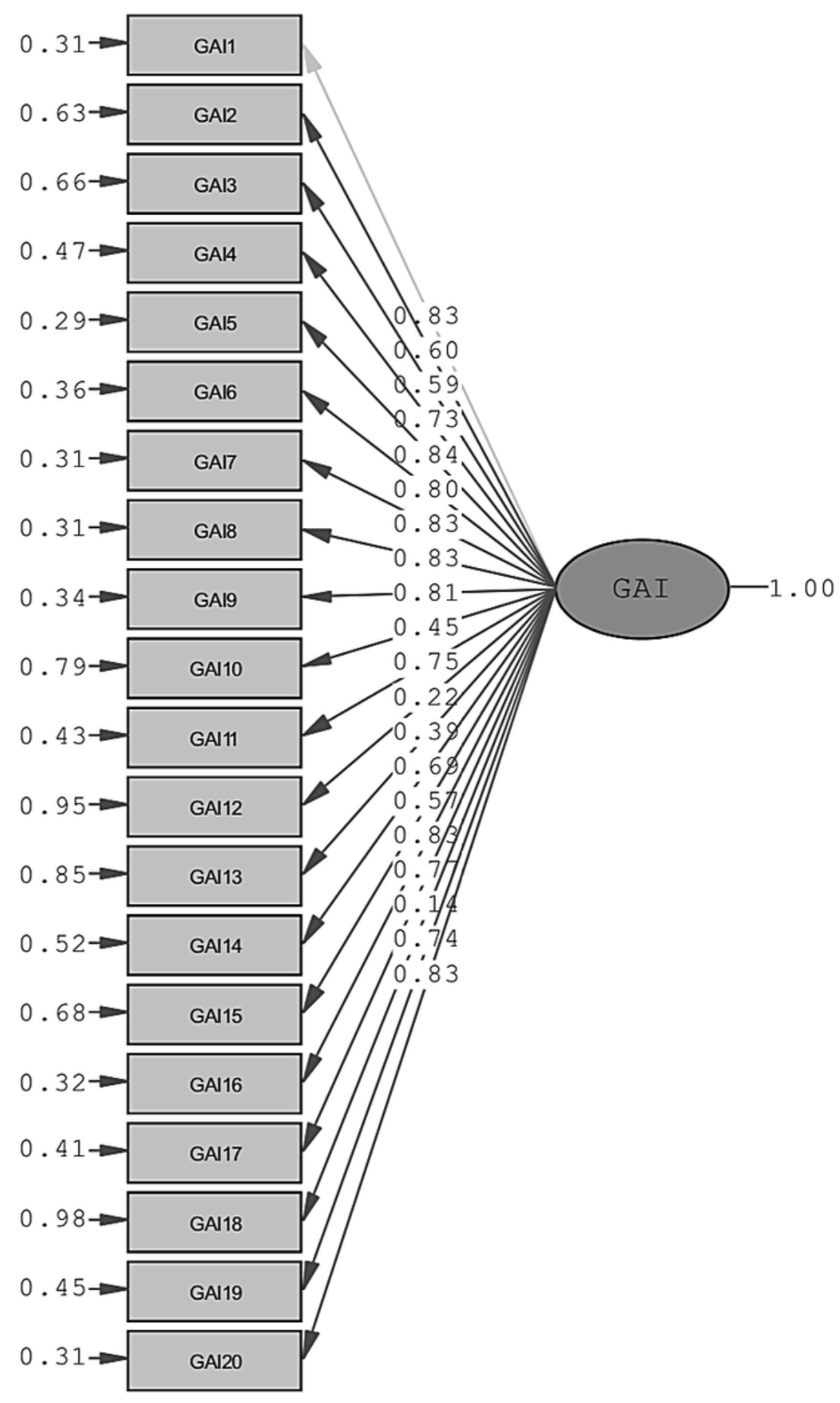

\section{Figure 1}

Preliminary model of the confirmatory factor analysis 


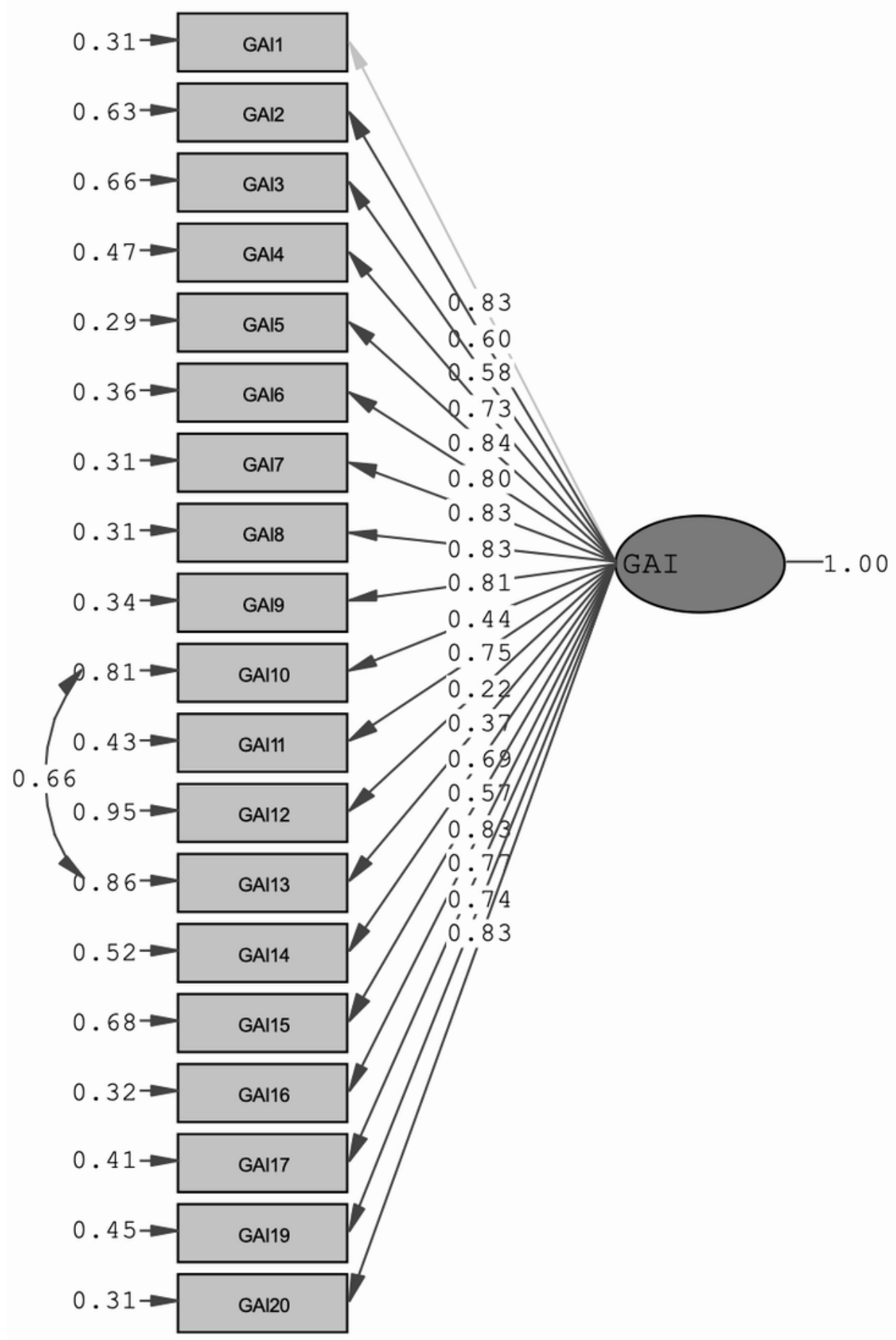

Figure 2

Final model of the confirmatory factor analysis

\section{Supplementary Files}

This is a list of supplementary files associated with this preprint. Click to download. 
- Appendix1.docx

Page 19/19 